\title{
Adjonction de vitamines aux denrées alimentaires et modalités de communication
}

\author{
Marianne DESSEN-MUGNIOT \\ Direction générale de la concurrence, \\ consommation et répression des fraudes \\ (DGCCRF), Bureau D3 - Secteur \\ Nutrition, 59 boulevard Vincent Auriol \\ 75703 Paris Cedex 13, France
}

\begin{abstract}
Regulation (EC) No 1925/2006 of 20 December 2006 on the addition of vitamins, minerals and certain other substances to foods came into force on 1 July 2007 and renders void certain provisions of Decree No. 2006/1264 of 16 October 2006. This text changes significantly how to approach the issue of current food fortification, hitherto subject to prior authorization. This article aims to review the conditions under which stakeholders can use the addition, and how the communication on packaging is possible, related to the presence of vitamins in food.
\end{abstract}

Key words: vitamins, fortification, RDA, nutrition claims, health claims

\section{Contexte et cadre réglementaire national}

L'enrichissement des aliments courants en vitamines, substances minérales et autres substances était auparavant soumis à la procédure d'autorisation définie par l'article $1^{\text {er }}$ du décrêt modifié du 15 avril 1912. La Cour de justice des Communautés européennes (CJCE) ayant condamné la France pour entrave à la libre circulation des marchandises du fait de l'inadaptation de cet article au droit communautaire, il a fallu revoir cette procédure. Les autorités françaises ont donc été contraintes de prendre des mesures adaptées, ce qui a donné lieu à la publication, le 17 octobre 2006, du décret $n^{\circ}$ 2006-1264 relatif aux vitamines, substances minérales et autres substances employées dans la fabrication des denrées alimentaires ${ }^{1}$.

Ce décret a introduit deux procédures d'autorisation de mise sur le marché des denrées auxquelles sont ajoutés des vitamines, minéraux et autres substances, selon que le principe de libre

\footnotetext{
${ }^{1}$ http://www.legifrance.gouv.fr/affichTexte. do?cidTexte=JORFTEXT000000817726\&dateTexte $=$
}

circulation trouve ou non à s'appliquer (articles 2 et 3). Son annexe 1 fixe la liste des vitamines et minéraux pouvant faire l'objet d'une demande d'autorisation d'emploi dans les denrées alimentaires. La liste des substances autres que vitamines et substances minérales $n^{\prime}$ est pas établie a priori. En revanche, le décret prévoit qu'un arrêté interministériel rende publique la liste des vitamines, minéraux et autres substances dont l'adjonction a été autorisée au cas par cas, et les conditions afférentes à cette autorisation d'emploi (matrice alimentaire concernée par l'enrichissement, teneurs minimales et maximales, mentions d'étiquetage, etc.), afin de permettre aux opérateurs concurrents d'accéder aux mêmes droits que le demandeur qui a, lui, bénéficié de la primauté de l'enrichissement.

\section{En 2007, I'Union européenne se dote d'un dispositif complet}

Le dispositif mis en place par la Commission européenne repose sur la publication de deux règlements, qui tendent à modifier, de manière notable, la notion d'enrichissement telle qu'entendue jusqu'alors par les autorités françaises.
En effet, le règlement (CE) $n^{\circ} 1925 /$ 2006 du 20 décembre 2006 concernant l'adjonction de vitamines, de minéraux et de certaines autres substances aux denrées alimentaires ${ }^{2}$, dont l'objectif est de s'assurer que l'adjonction de vitamines et minéraux conduit à la commercialisation de produits sûrs, ne fait pas, contrairement aux dispositions françaises en vigueur jusque-là, de cas particuliers en fonction des objectifs recherchés de l'adjonction : les mêmes règles (notamment, limites maximales et autres restrictions ou interdictions) s'appliquent donc aux denrées additionnées de vitamines et minéraux que l'adjonction soit réalisée dans un but d'enrichissement, de restauration ou de recherche d'équivalence nutritionnelle.

En outre, la publication simultanée du règlement (CE) $n^{\circ} 1924 / 2006$ relatif aux allégations nutritionnelles et de santé portant sur les denrées alimentaires $^{3}$ complète ce dispositif dont le

\footnotetext{
${ }^{2}$ http://eur-lex.europa.eu/LexUriServ/LexUriServ.do?uri=OJ:L:2006:404:0026:0038:FR: PDF

${ }^{3}$ http://eur-lex.europa.eu/LexUriServ/LexUriServ.do?uri=OJ:L:2007:012:0003:0018:FR: PDF
} 99-103. doi : 10.1684/ocl.2011.0371 
second objectif est d'assurer une information loyale du consommateur, ne l'induisant pas en erreur sur les bénéfices qu'il est supposé en attendre (notamment en fixant une quantité minimale de nutriments ou substances dans I'aliment dit " enrichi ", en établissant des seuils applicables aux allégations nutritionnelles et une liste positive des allégations de santé autorisées...).

\section{Le dispositif européen a modifié le cadre national en vigueur}

De manière plus précise, le règlement (CE) $n^{\circ} 1925 / 2006$, entré en application le $1^{\text {er }}$ juillet 2007, vise notamment à rendre licite l'enrichissement des denrées alimentaires (à l'exception des compléments alimentaires pour lesquels existent des dispositions spécifiques dans le cadre de la directive 2002/46), en vitamines et minéraux dont la liste est établie en annexe I (A, D, E, K, B1, B2, Niacine, Acide pantothénique, B6, B9, B12, Biotine, $C$ ), et sous les formes énoncées en annexe II (considérées comme inoffensives et biodisponibles) ${ }^{4}$. En vertu de l'article 17.1 du règlement, pris sur la base du principe de libre-circulation des marchandises, les denrées alimentaires auxquelles ont été ajoutées des vitamines et minéraux sous des formes d'apport autorisées dans d'autres États membres de I'Union européenne et soumises à la Commission au titre de I'article 17, peuvent, dans l'attente de leur évaluation par l'Autorité européenne de sécurité des aliments (AESA), être commercialisées sur le marché français. Ces éléments figurent dans le registre communautaire sur l'adjonction de vitamines, minéraux et certaines autres substances, établi conformément à l'article 9 du règlement ${ }^{5}$.

\footnotetext{
${ }^{4}$ Il convient de noter que les dispositions de ce règlement ne visent que l'adjonction " directe " de vitamines et minéraux, et que l'enrichissement par le biais d'autres moyens, tels que l'utilisation d'un ingrédient contenant " naturellement " une quantité notable de vitamines et/ou minéraux (par exemple : lithotamme, présentant naturellement une teneur élevée en calcium), n'est pas explicitement visé par ce texte.

${ }^{5}$ http://ec.europa.eu/food/food/labellingnutrition/vitamins/comm_reg_en.pdf
}

Ce règlement rend en conséquence caduques les dispositions du décret $n^{\circ}$ 2006-1264 en ce qu'elles concernent les vitamines et minéraux énumérés à I'annexe I du règlement 1925/2006, en particulier la procédure d'autorisation préalable à la mise sur le marché. Ainsi, depuis le $1^{\text {er }}$ juillet 2007, la commercialisation de ces produits peut être envisagée sur le territoire français sans attendre l'aval de l'administration, dès lors que les produits sont conformes aux dispositions du règlement (CE) $n^{\circ} 1925 / 2006$.

De la même manière, les arrêtés autorisant l'emploi de certaines vitamines et/ ou minéraux dans des denrées alimentaires courantes, anciennement pris sur la base du décret du 15 avril 1912 ne sont plus applicables (en particulier l'arrêté du 8 octobre 2004 relatif à l'emploi de vitamine D3 dans les huiles végétales, certaines dispositions de l'arrêté du 20 juillet 1977 sur les produits diététiques et de régime, notamment celles du chapitre IV relatives aux produits de régime destinés aux régimes qui nécessitent un apport lipidique particulier).

Le règlement (CE) n 1925/2006 précise les conditions selon lesquelles les vitamines et minéraux peuvent être ajoutés aux denrées alimentaires.

- Des teneurs minimales en nutriments sont prévues pour éviter d'induire le consommateur en erreur.

L'article 6.6 du règlement prévoit que l'adjonction d'une vitamine ou d'un minéral dans un aliment doit aboutir à la présence, dans l'aliment, d'au moins une "quantité significative » de cette vitamine ou de ce minéral. La notion de " quantité significative » fait référence aux termes employés à l'annexe de la directive 90/496 relative à l'étiquetage nutritionnel $^{6}$. II en ressort que toute adjonction de vitamines ou de minéraux doit conduire à la présence dans le produit fini d'au moins $15 \%$ des apports journaliers recommandés (AJR), tels qu'ils y sont définis, pour $100 \mathrm{~g}, 100 \mathrm{~mL}$ ou par emballage lorsque I'emballage ne contient qu'une portion.

\footnotetext{
${ }^{6}$ http://eur-lex.europa.eu/LexUriServ/LexUriServ.do?uri=CELEX:31990L0496:FR:HTML

${ }^{7}$ http://eur-lex.europa.eu/LexUriServ/LexUriServ.do?uri=OJ:L:2008:285:0009:0012:FR: PDF
}

La directive 90/496/CEE a été modifiée par la directive 2008/100/CE ${ }^{7}$, transposée en droit français par l'arrêté du 24 février $2010^{8}$. Les valeurs des AJR ont été revues pour certains nutriments et la liste a été complétée de telle manière qu'à ce stade le cadre communautaire prévoit des AJR pour I'ensemble des vitamines et minéraux pouvant être ajoutés aux denrées alimentaires. Les valeurs sont reprises dans le tableau 1 .

- Des teneurs maximales sont prévues pour éviter des absorptions excessives préjudiciables à la santé des consommateurs.

Afin d'assurer la sécurité du consommateur, le règlement prévoit également qu'en cas d'adjonction de vitamines et/ou minéraux dans une denrée, la quantité totale de la vitamine ou du minéral présent, à quelque fin que ce soit, dans les aliments mis en vente ne dépasse pas les teneurs maximales établies dans le cadre de ce texte. Bien que le règlement prévoie que la Commission aurait du faire une proposition avant le 31 janvier 2009, ces valeurs ne sont pas encore établies.

De manière consensuelle, la détermination des quantités maximales s'appuie sur le principe général de l'analyse des risques adaptée au cas des nutriments, rappelés à l'article 6 du règlement 1925/ $2006^{9}$. L'évaluation des risques conduit tout d'abord à classer les nutriments en fonction du risque de dépassement de la limite de sécurité. Cette classification est obtenue en comparant la limite de sécurité (lorsqu'elle a pu être établie) avec les apports alimentaires, la différence obtenue correspondant à la marge disponible pour la supplémentation. À ce titre, les deux principales organisations européennes regroupant les fabricants de compléments alimentaires (ERNA et EHPM), également relayées par la Confédération européenne des indus-

\footnotetext{
${ }^{8}$ Modifiant I'arrêté du 3 décembre 1993 portant application du décret $n^{\circ}$ 93-1130 du 27 septembre 1993 concernant l'étiquetage relatif aux qualités nutritionnelles des denrées alimentaires

${ }^{9}$ Selon les principes introduits à l'article 6 du règlement, ces teneurs maximales doivent être fixées en tenant compte des limites supérieures de sécurité, des apports en vitamines et minéraux en provenance d'autres sources alimentaires et des apports de référence recommandés pour la population.
} 
Tableau 1. Vitamines et minéraux pouvant être ajoutés et/ou et déclarés, et leurs apports journaliers recommandés (AJR) tels que précisés dans la directive 2008/100

\begin{tabular}{|c|c|c|c|}
\hline Vitamine $\mathrm{A}(\mu \mathrm{g})$ & 800 & Potassium (mg) & 2000 \\
\hline Vitamine $\mathrm{D}(\mu \mathrm{g})$ & 5 & Chlorure (mg) & 800 \\
\hline Vitamine $\mathrm{E}(\mathrm{mg})$ & 12 & Calcium (mg) & 800 \\
\hline Vitamine $\mathrm{K}(\mu \mathrm{g})$ & 75 & Phosphore (mg) & 700 \\
\hline Vitamine C (mg) & 80 & Magnésium (mg) & 375 \\
\hline Thiamine (mg) & 1,1 & Fer $(\mathrm{mg})$ & 14 \\
\hline Riboflavine (mg) & 1,4 & Zinc (mg) & 10 \\
\hline Niacine (mg) & 16 & Cuivre (mg) & 1 \\
\hline Vitamine B6 (mg) & 1,4 & Manganèse (mg) & 2 \\
\hline Folate $(\mu \mathrm{g})$ & 200 & Fluor (mg) & 3.5 \\
\hline Vitamine B12 $(\mu \mathrm{g})$ & 2,5 & Sélénium $(\mu g)$ & 55 \\
\hline Biotine (mg) & 50 & Chrome $(\mu \mathrm{g})$ & 40 \\
\hline \multirow{2}{*}{ Acide Pantothenique (mg) } & \multirow{2}{*}{6} & Molybdène $(\mu \mathrm{g})$ & 50 \\
\hline & & lode $(\mu \mathrm{g})$ & 150 \\
\hline
\end{tabular}

tries alimentaires (CIAA), ont proposé des modèles conduisant à catégoriser les nutriments en trois groupes, en comparant la limite de sécurité aux apports alimentaires du $97,5^{\mathrm{e}}$ percentile de la population : les vitamines pour lesquelles il n'existe pas de risque de dépassement des limites de sécurité (B1, B2, B12, biotine, acide pantothénique, $\mathrm{K}^{10}$ ), les vitamines pour lesquelles il existe un faible risque de dépassement $(E, D, B 6$, nicotinamide, B9), les vitamines pour lesquelles le risque de dépassement des limites de sécurité est élevé ( $A$, bêtacarotène).

Dans un deuxième temps, des valeurs maximales de supplémentation sont établies par le gestionnaire du risque, en tenant compte des résultats de l'évaluation des risques et des incertitudes soulevées.

Comme a pu le rappeler la Cour de justice des Communautés européennes à plusieurs reprises, "l'évaluation du risque (...) a pour objet I'appréciation du degré de probabilité des effets néfastes de I'adjonction de certaines substances nutritives aux denrées alimentaires pour la santé humaine et de la gravité de ces effets potentiels ». Dans le cas présent, il

\footnotetext{
${ }^{10}$ Compte tenu de la prévalence élevée de sujets suivant un traitement anti-coagulant par AVK, l'Anses recommande une extrême prudence en matière d'enrichissement des aliments courants en vitamine $\mathrm{K}$ (avis du 13 juin 2008, disponible sur http://www.afssa. fr/Documents/NUT2007sa0315.pdf). C'est pourquoi, le classement de la vitamine $\mathrm{K}$ dans ce groupe est sujet à discussion.
}

$s^{\prime}$ agit donc de déterminer pour chaque nutriment la dose acceptable dans les denrées alimentaires, au-delà de laquelle la survenue des dangers identifiés devient réelle et donc inacceptable pour le gestionnaire du risque.

Dans le cadre de cette réflexion, l'Agence nationale de sécurité sanitaire chargée de I'alimentation, de l'environnement et du travail (Anses) - anciennement Agence française de sécurité sanitaire des aliments (Afssa) - a été saisie par ses ministères de tutelle afin de proposer des teneurs maximales pouvant servir de base aux autorités françaises dans le cadre des négociations communautaires. Ses travaux se fondent sur des simulations qui consistent à évaluer les risques de dépassement des limites de sécurité en fonction de divers scénarios de supplémentation (à la fois par le biais des compléments alimentaires et de I'alimentation enrichie) utilisant les données de consommation françaises les plus récentes (étude INCA 2). Les résultats sont publiés sur le site internet de I'Agence ${ }^{11}$.

Depuis 2007, de nombreux échanges ont eu lieu entre les États membres et la Commission européenne, qui n'ont pas encore abouti. Une proposition de la Commission est attendue dans le courant de l'année 2011 (toute information rendue publique sur ce sujet devrait être disponible sur le site internet de la Commission) ${ }^{12}$.

\footnotetext{
11 http://www.afssa.fr/Documents/ NUT2007sa0315t2.pdf
}

Dans l'attente d'une position définitive au niveau communautaire et conformément aux principes du règlement (CE) 178/2002 établissant les principes généraux de la législation alimentaire ${ }^{13}$, les opérateurs peuvent proposer des niveaux d'enrichissement qui conduisent à la fabrication de denrées alimentaires sûres et non préjudiciables à la santé des consommateurs. II leur appartient, en tant que professionnels, de s'assurer de la sécurité de leurs produits en s'appuyant sur l'ensemble des informations en leur possession, notamment les données scientifiques généralement acceptées (encadré 1).

\section{Quelles exigences en matière d'étiquetage ?}

\section{Un étiquetage nutritionnel complet}

Le règlement (CE) $n^{\circ}$ 1925/2006 impose que les denrées auxquelles ont été ajoutées des vitamines et minéraux comportent un étiquetage nutritionnel de groupe 2 (Énergie, protéines, glucides, dont les sucres, lipides, dont les acides gras saturés, fibres, sodium), ainsi que les quantités totales des vitamines et des minéraux qui ont été ajoutés à I'aliment.

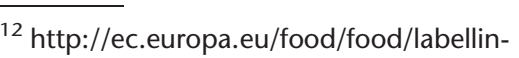

gnutrition/vitamins/index_en.htm

${ }^{13}$ http://eur-lex.europa.eu/LexUriServ/ LexUriServ.do?uri=CONS-

LEG:2002R0178:20060428:FR:PDF
} 


\section{Encadré 1}

\section{L'exemple de la vitamine $A$}

L'Anses montre, dans son avis daté du 27 janvier $2009^{24}$, qu'un enrichissement des aliments courants en vitamine A (à hauteur de $120 \mu \mathrm{g}$ pour $100 \mathrm{kcal}$ ) associé à une consommation de compléments alimentaires en contenant $800 \mu \mathrm{g}$, double le pourcentage de personnes dépassant la limite de sécurité. À ce titre, elle recommande de ne pas enrichir les aliments courants en vitamine A (et de maintenir la teneur maximale des compléments alimentaires à $800 \mu \mathrm{g}$ ). Par ailleurs, dans ce même avis, elle rappelle que les données françaises d'apport démontrent l'absence de carence ou de déficit en vitamine A : " Des publications confirment que la prévalence d'apports inadéquats en vitamine $A$ totale est très faible chez les adultes français et bien moindre que pour d'autres vitamines ou minéraux (Touvier et al., 2005, de Lauzon et al., 2004) et qu'on ne peut identifier de groupes à risque de déficit en vitamine $A$ totale dans la population française. "

En outre, dans un précédent avis daté du 13/07/2007 sur l'emploi de vitamine A à $800 \mathrm{mg} / 100 \mathrm{~g}$ dans margarines et $\mathrm{MG}$ à tartiner, l'Anses indique également que les niveaux de consommation au P97.5 chez les enfants (avant enrichissement) sont supérieurs à la limite de sécurité définie par le Comité scientifique de I'alimentation humaine (CSAH, 2002). De ce fait, et dans l'attente de dispositions harmonisées au niveau européen, les opérateurs ont été invités par les pouvoirs publics à prendre des mesures permettant de s'assurer que les margarines et les matières grasses additionnées de vitamine A ne seront pas consommées par les enfants.

\section{Des allégations nutritionnelles et de santé encadrées}

Avant juillet 2007, les allégations de santé étaient encadrées par des dispositions très générales d'obligation de publicité non trompeuse et d'interdiction de faire état de prévention, traitement ou guérison de maladie (dispositions de la directive 2000/13 sur l'étiquetage et la publicité et du Code de la consommation). En quelques années, le créneau de la " nutrition-santé » s'est imposé dans le paysage agroalimentaire européen comme l'une des voies d'innovation majeure, s'accompagnant d'une intensification de la communication à visée " santé ». Face à la profusion en Europe de ces allégations sur les emballages et la publicité des denrées alimentaires, I'Union européenne a choisi de se doter $d^{\prime}$ 'un dispositif spécifique. Le règlement (CE) $n^{\circ} 1924 / 2006$ entré en vigueur en juillet 2007 a un double objectif : assurer un haut niveau de protection des consommateurs (toute allégation doit être fondée sur des justifications scientifiques bien établies) tout en préservant les conditions d'une concurrence loyale entre les industries agroalimentaires.

La notion d'allégation est définie à l'article 2-2, de façon très large. Une allégation est, par nature, volontaire. Tout message ou toute représentation qui affirme, suggère ou implique qu'une denrée alimentaire possède des caractéristiques listes. Dès l'établissement de ces listes, les allégations nouvelles sont soumises particulières est concerné. Cela inclut des représentations sous forme d'images, Le règlement distingue plusieurs types d'allégations (encadré 2).

Depuis son entrée en application, reau rèlement induit des mod prévoit l'établissement de listes positives $d$ allégations nutritionnelles et de santé ainsi qu'un système d'autorisation préalable pour toute allégation ajoutée à ces à autorisation préalable avec une centralisation des demandes à la Commission européenne.

Ainsi, seules les allégations nutritionnelles citées dans l'annexe du règlement sont autorisées aux conditions qui y sont spécifiées.

Contrairement aux dispositions anciennement en vigueur, qui rendaient obligatoire une description précise de la denrée (dénomination descriptive, précisant notamment le recours à un enrichissement), à compter de la publication du règlement (CE) $n^{\circ} 1924 / 2006$, toute mention relative à la présence (présence " naturelle » ou par le biais d'une addition) de vitamines ou de minéraux, que ce soit dans la dénomination de vente ou ailleurs sur l'étiquetage, doit désormais être considérée comme une allégation nutritionnelle et en conséquence être formulée dans le respect des dispositions dudit règlement. En particulier, comme le précise son considérant 21 , dans I'hypothèse où la dénomination de vente est complétée par des mentions du type "[vitamine] ajoutée", "...enrichie en [vitamine] ", "avec une teneur en [vitamine] ..." " " à teneur garantie en [vitamine]", "à teneur restaurée en [vitamine] ", ou " contient [vitamine]", ces mentions doivent être considérées comme des allégations soumises aux conditions de l'allégation " source de... ".

À titre complémentaire, l'annexe du règlement listant les allégations nutritionnelles autorisées a été complétée par 5 allégations relatives aux acides gras (source et riche en acides gras

\section{Encadré 2}

\section{Les principaux types d'allégations}

L'allégation nutritionnelle est une mention quantitative mettant en avant l'absence ou la présence, en plus ou moins grande quantité, de certains composants des aliments ou de leur apport énergétique : " pauvre en sucres ", " $0 \%$ de matières grasses ", " allégé " ou encore " riche en vitamine $D$ ".

L'allégation de santé fait le lien entre aliment et un paramètre de santé. II décrit l'impact possible du produit sur l'état de santé : "Par son apport en calcium, le produit $\mathrm{X}$ contribue à renforcer les os ». Le règlement distingue :

- les allégations de santé faisant référence au développement et à la santé des enfants ou à la réduction d'un risque de maladie (art. 14), dont les procédures d'examen et d'autorisation sont plus longues;

- Les autres allégations de santé (art. 13), au sein desquelles :

¿ les allégations génériques ( $(1)$ déjà utilisées avant l'entrée en vigueur du texte. Elles reposent sur des données scientifiques bien établies et sont bien comprises par le consommateur ;

to des allégations innovantes ( $(5)$ reposant sur des données nouvelles. 
oméga 3 ; riche en acides gras insaturés, riche en acides gras mono-insaturés, riche en acides gras polyinsaturés), et les discussions se poursuivent au niveau communautaire pour faire évoluer cette liste (révision des conditions applicables à certaines allégations, inclusion de nouvelles allégations) ${ }^{14}$.

Par ailleurs, le règlement encadre également l'emploi d'allégations de santé qu'il définit comme "toute allégation qui affirme, suggère ou implique l'existence d'une relation entre, d'une part, une catégorie de denrées alimentaires, une denrée alimentaire ou I'un de ses composants et, d'autre part, la santé ". Les allégations de santé se partagent entre celles relevant de l'article 14 (allégations faisant référence à la réduction du risque de maladie ainsi quau développement et à la santé infantiles), pour lesquelles les procédures d'examen et d'autorisation sont plus longues, et celles relevant de l'article 13, souvent appelées "allégations de santé fonctionnelles".

Le règlement $(C E) n^{\circ}$ 1924/2006 prévoit l'établissement, au 31 janvier 2010, d'une liste positive d'allégations de santé "génériques " autorisées. Cette liste doit permettre à tout industriel de I'agroalimentaire d'utiliser sur leurs denrées alimentaires des allégations de santé "fonctionnelles " présentes dans la liste, sous réserve du respect des conditions d'utilisation associées. L'établissement de cette liste, qui constitue un important travail, a débuté en 2007 en recensant, dans chaque État membre, les allégations répondant aux critères établis dans le règlement. Sur la base des 27 listes nationales, la Commission et I'AESA ont ensuite élaboré un projet de liste communautaire d'environ 4600 allégations. En juillet 2008, ces allégations ont été soumises à l'AESA en vue d'examiner la validité scientifique des demandes.

L'évaluation scientifique par I'AESA, et donc l'adoption de cette liste par les Etats membres ont été considérablement complexifiées et ralenties par la quantité d'allégations déposées. Par conséquent, le délai d'adoption de la liste complète au 31 janvier 2010 prévu par le règlement

\footnotetext{
${ }^{14}$ Voir les modifications récentes sur le site internet de la Commission : http://ec.europa. eu/food/food/labellingnutrition/claims/ nutrition_claims_en.htm
}

(CE) n 1924/2006 n'a pas été respecté. À ce jour,quatre lots d'avis scientifiques rendus par I'AESA ont été publiés, respectivement en octobre 2009 , février 2010, octobre 2010 et avril 2011. La Commission a demandé à I'AESA de publier la totalité de ses avis concernant les nutriments et substances (autres que les plantes) pour la fin du mois de juin 2011, et envisage d'adopter, sur la base de ces avis, une première liste positive d'allégations de santé autorisées pour la fin de l'année 2011.

Concernant plus particulièrement les vitamines liposolubles, début 2011, 15 allégations de santé "génériques " ont fait l'objet d'un avis favorable de l'AESA. L'Agence a en effet considéré que les preuves scientifiques disponibles permettaient de conclure que :

- la vitamine A contribuait au fonctionnement normal du système immunitaire $^{15}$, à une différenciation cellulaire normale $^{16}$, au maintien en bon état de la peau et des muqueuses ${ }^{16}$, ainsi qu'au maintien $d^{\prime}$ une bonne vision ${ }^{16}$ (en association avec du bêta-carotène) ; - la vitamine E contribuait à la protection des constituants cellulaires contre l'oxydation (propriétés anti-oxydantes) ${ }^{17}$; - la vitamine D contribuait au maintien normal des os (et que dans cette fonction, elle était nécessaire en association avec le calcium ${ }^{18}$ ) et des dents ${ }^{19}$, à une absorption/utilisation normale du calcium et du phosphore et au maintien du taux de calcium dans le sang ${ }^{20}$, au fonctionnement normal du système immunitaire et de la réponse inflammatoire ${ }^{20}$, au maintien de la fonction musculaire normale ${ }^{21}$;

- la vitamine $\mathrm{K}$ contribuait au maintien normal des os (fonction en particulier reconnu pour la menaquinone-7), ainsi qu'au maintien de la coagulation sanguine normale (K1 et K2) ${ }^{21}$;

\footnotetext{
${ }^{15}$ http://www.efsa.europa.eu/en/efsajournal/doc/1221.pdf

${ }^{16}$ http://www.efsa.europa.eu/en/efsajournal/doc/1754.pdf

17 http://www.efsa.europa.eu/en/efsajournal/doc/1816.pdf

${ }^{18}$ http://www.efsa.europa.eu/en/efsajournal/doc/1272.pdf

${ }^{19}$ http://www.efsa.europa.eu/en/efsajournal/doc/1227.pdf

${ }^{20}$ http://www.efsa.europa.eu/en/efsajournal/doc/1468.pdf
}

- concernant les allégations de santé relevant de l'article 14, les dossiers de demande d'autorisation doivent être établis selon les lignes directrices publiées par l'AESA ${ }^{22}$ et transmis à la DGCCRF - bureau D3 qui mène, avant transmission à I'AESA, un examen juridique du dossier en s'assurant de sa conformité au règlement. À l'issue de l'avis de I'AESA, la Commission soumet aux États membres un projet de décision d'autorisation ou de refus d'autorisation de l'allégation. Les décisions sont prises par voie de règlements, qui sont adoptés selon les procédures de Comitologie (avec contrôle du Parlement) et publiés au Journal officiel de l'Union européenne.

À ce stade, seules deux demandes relatives à des vitamines liposolubles ont fait l'objet d'avis favorables de I'AESA, suivis de décisions d'autorisation de la Commission européenne : ces allégations concernent le rôle de la vitamine $D$, seule et en association avec le calcium, dans la croissance et le développement osseux normaux des enfants ${ }^{23}$.

\section{Conclusion}

La santé est devenue un argument de vente majeur pour I'industrie agro-alimentaire. Ainsi, environ trois quarts des produits alimentaires vendus en France affichent une allégation nutritionnelle ou de santé. À la fois consciente des enjeux économiques et soucieuse de protéger le consommateur, I'Union européenne s'est dotée d'un dispositif complet, dans le double objectif $d$ 'assurer la sécurité des consommateurs et leur information loyale. À terme, elle doit également permettre aux industriels de valoriser des produits pour lesquels les effets sur la santé sont justifiés, et ainsi soutenir l'innovation. Sa mise en œuvre progressive est le reflet de la complexité et de I'envergure du travail à effectuer.

\footnotetext{
${ }^{21}$ http://www.efsa.europa.eu/en/efsajournal/doc/1228.pdf

${ }^{22}$ Scientific and technical guidance for the preparation and presentation of the application for authorisation of a health claim - 23 juillet 2007 - http://www.efsa.europa.eu/ EFSA/Scientific_Opinion/nda_op_ej530_guidance_\%20health_claim_en.pdf,0.pdf ${ }^{23}$ Commission Regulation (EC) No 983/ 2009 of 21/10/2009 : http://eur-lex.europa. eu/LexUriServ/LexUriServ.do?uri=OJ:L:2009: 277:0003:0012:FR:PDF
} 